\title{
Stereotactic radiosurgery for intracranial hemangioblastomas: a retrospective international outcome study
}

\author{
Hideyuki Kano, MD, PhD, ${ }^{1}$ Takashi Shuto, MD, ${ }^{2}$ Yoshiyasu Iwai, MD, PhD, ${ }^{3}$ \\ Jason Sheehan, MD, PhD, ${ }^{4}$ Masaaki Yamamoto, MD, ${ }^{5}$ Heyoung L. McBride, MD, ${ }^{6}$ \\ Mitsuya Sato, MD, PhD, ${ }^{7}$ Toru Serizawa, MD, PhD, ${ }^{8}$ Shoji Yomo, MD, PhD, ${ }^{9}$ \\ Akihito Moriki, MD, PhD, ${ }^{10}$ Yukihiko Kohda, MD, PhD, ${ }^{11}$ Byron Young, MD, ${ }^{12}$ \\ Satoshi Suzuki, MD, PhD, ${ }^{13}$ Hiroyuki Kenai, MD, PhD, ${ }^{14}$ Christopher Duma, MD, ${ }^{15}$ \\ Yasuhiro Kikuchi, MD, PhD, ${ }^{16}$ David Mathieu, MD, ${ }^{17}$ Atsuya Akabane, MD, PhD, ${ }^{18}$ \\ Osamu Nagano, MD, ${ }^{19}$ Douglas Kondziolka, MD, ${ }^{20}$ and L. Dade Lunsford, MD ${ }^{1}$
}

1Departments of Neurological Surgery, University of Pittsburgh, Pennsylvania; ${ }^{2}$ Yokohama Rosai Hospital, Yokohama; ${ }^{3}$ Osaka City General Hospital, Osaka; ${ }^{5}$ Katsuta Hospital Mito GammaHouse, Hitachinaka; ${ }^{7}$ Kitanihon Hospital, Gosen; ${ }^{8}$ Tsukiji Neurological Clinic, Tokyo; ${ }^{9}$ Saitama Gamma Knife Center, Saitama; ${ }^{10}$ Mominoki Hospital, Kochi; ${ }^{11}$ Asanogawa General Hospital, Kanazawa; ${ }^{13}$ Steel Memorial Yawata Hospital, Kitakyushyu; ${ }^{14}$ Nagatomi Neurosurgical Hospital, Oita; ${ }^{16}$ Southern Tohoku Hospital, Koriyama; ${ }^{18 N T T}$ Kanto Hospital, Tokyo; ${ }^{19} \mathrm{Chiba}$ Cardiovascular Center, Ichihara, Japan; ${ }^{4}$ University of Virginia, Charlottesville, Virginia; ${ }^{6}$ Barrow Neurological Institute, Phoenix, Arizona; ${ }^{12}$ University of Kentucky, Lexington, Kentucky; ${ }^{15} \mathrm{Hoag}$ Hospital, Newport Beach, California; ${ }^{17 U n i v e r s i t e ́ ~ d e ~ S h e r b r o o k e, ~ C e n t r e ~ d e ~ R e c h e r c h e ~ C l i n i q u e ~ E ́ t i e n n e-L e B e l, ~ S h e r b r o o k e, ~}$ Quebec, Canada; and ${ }^{20 N e w ~ Y o r k ~ U n i v e r s i t y ~ L a n g o n e ~ M e d i c a l ~ C e n t e r, ~ N e w ~ Y o r k, ~ N e w ~ Y o r k ~}$

OBJECT. The purpose of this study was to evaluate the role of stereotactic radiosurgery (SRS) in the management of intracranial hemangioblastomas.

METHODS. Six participating centers of the North American Gamma Knife Consortium and 13 Japanese Gamma Knife centers identified 186 patients with 517 hemangioblastomas who underwent SRS. Eighty patients had 335 hemangioblastomas associated with von Hippel-Lindau disease (VHL) and 106 patients had 182 sporadic hemangioblastomas. The median target volume was $0.2 \mathrm{~cm}^{3}$ (median diameter $7 \mathrm{~mm}$ ) in patients with VHL and $0.7 \mathrm{~cm}^{3}$ (median diameter 11 $\mathrm{mm}$ ) in those with sporadic hemangioblastoma. The median margin dose was $18 \mathrm{~Gy}$ in VHL patients and 15 Gy in those with sporadic hemangioblastomas.

RESULTS. At a median of 5 years (range $0.5-18$ years) after treatment, 20 patients had died of intracranial disease progression and 9 patients had died of other causes. The overall survival after SRS was $94 \%$ at 3 years, $90 \%$ at 5 years, and $74 \%$ at 10 years. Factors associated with longer survival included younger age, absence of neurological symptoms, fewer tumors, and higher Karnofsky Performance Status. Thirty-three (41\%) of the 80 patients with VHL developed new tumors and $17(16 \%)$ of the106 patients with sporadic hemangioblastoma had recurrences of residual tumor from the original tumor. The 5 -year rate of developing a new tumor was $43 \%$ for $\mathrm{VHL}$ patients, and the 5 -year rate of developing a recurrence of residual tumor from the original tumor was $24 \%$ for sporadic hemangioblastoma patients. Factors associated with a reduced risk of developing a new tumor or recurrences of residual tumor from the original tumor included younger age, fewer tumors, and sporadic rather than VHL-associated hemangioblastomas. The local tumor control rate for treated tumors was $92 \%$ at 3 years, $89 \%$ at 5 years, and $79 \%$ at 10 years. Factors associated with an improved local tumor control rate included VHL-associated hemangioblastoma, solid tumor, smaller tumor volume, and higher margin dose. Thirteen patients (7\%) developed adverse radiation effects (ARE) after SRS, and one of these patients died due to ARE.

ABBREVIATIONS ARE = adverse radiation effects; KPS = Karnofsky Performance Status; NAGKC $=$ North American Gamma Knife Consortium; SRS $=$ stereotactic radiosurgery; UPMC = University of Pittsburgh Medical Center; UVA = University of Virginia; VHL = von Hippel-Lindau disease.

ACCOMPANYING EDITORIAL See pp 1466-1468. DOI: 10.3171/2015.2.JNS15252.

SUBMITTED July 24, 2013. ACCEPTED October 27, 2014.

INCLUDE WHEN CITING Published online March 27, 2015; DOI: 10.3171/2014.10.JNS131602.

DISCLOSURE Drs. Lunsford and Kondziolka are consultants for Elekta AB and Dr. Lunsford is a stockholder. 
CONCLUSIONS. When either sporadic or VHL-associated tumors were observed to grow on serial imaging studies, SRS provided tumor control in $79 \%-92 \%$ of tumors.

http://thejns.org/doi/abs/10.3171/2014.10.JNS131602

KEY WORDS stereotactic radiosurgery; Gamma Knife; hemangioblastoma; von Hippel-Lindau disease

$\mathrm{H}$ EMANGIOBLASTOMAS are highly vascular but often well-circumscribed tumors of the central nervous system and are most often detected in the posterior fossa. ${ }^{3,26}$ Hemangioblastomas of the brain present both as sporadic lesions or as manifestations of von HippelLindau disease (VHL). ${ }^{5,6,23}$ Approximately 20\%-25\% of hemangioblastomas are associated with VHL. ${ }^{15}$ Sporadic hemangioblastomas occur predominantly in the cerebellum, whereas VHL-associated hemangioblastomas may develop in the cerebellum, brainstem, and spinal cord. ${ }^{12}$ Compared with sporadic hemangioblastomas, VHL-associated hemangioblastomas tend to present in younger patients. ${ }^{12}$ Patients with sporadic hemangioblastomas have a better overall prognosis, because patients with VHL tend to develop additional tumors over time. ${ }^{14}$

When feasible, resection of a symptomatic hemangioblastoma may be curative. However, either the vascularity of a tumor or a critical location may make a complete resection too risky, especially in patients with multiple tumors. In the past, fractionated radiation therapy has been performed for residual, recurrent, or surgically inaccessible tumors. ${ }^{22,24}$ During the past 25 years stereotactic radiosurgery (SRS) has been used as a minimally invasive primary, adjuvant, or salvage option for patients with hemangioblastomas. 2,4,11,13,16-18,20,25,28 The North American Gamma Knife Consortium (NAGKC) was established to evaluate outcomes of SRS for conditions that are relatively rare (e.g., chordoma, ${ }^{9}$ cluster headache,${ }^{10}$ glomus tumors ${ }^{21}$ ) and to facilitate prospective clinical trials. This report is based on retrospective data provided by 6 academic medical centers that participate in the NAGKC and 13 Japanese Gamma Knife centers. We analyzed the outcomes of SRS in patients with hemangioblastoma to better understand various prognostic features.

\section{Methods}

\section{Patient Population}

Between 1990 and 2010, 6 participating centers of the NAGKC and 13 participating Japanese Gamma Knife centers identified 186 patients with 517 hemangioblastomas, all of which were treated with SRS. The following Gamma Knife SRS centers had individual internal review board approvals for retrospective clinical outcome studies and submitted cases for this report: University of Pittsburgh Medical Center (UPMC, $\mathrm{n}=42$ ), University of Kentucky $(n=5)$, Hoag Hospital $(n=4)$, University of Sherbrooke $(n=3)$, University of Virginia (UVA, $n=16$ ), Barrow Neurological Institute $(n=11)$, Yokohama Rosai Hospital ( $n=23)$, NTT Kanto Hospital $(n=3)$, Katsuta Hospital Mito Gamma House $(n=13)$, Tsukiji Neurological Clinic $(n=6)$, Mominoki Hospital $(n=6)$, Southern Tohoku Hospital $(n=4)$, Steel Memorial Yawata Hospital $(n=5)$, Saitama Gamma Knife Center $(n=6)$, Osaka City
General Hospital $(\mathrm{n}=18)$, Ciba Cardiovascular Center ( $\mathrm{n}$ $=1)$, Kitanihon Hospital $(\mathrm{n}=10)$, Nagatomi Neurosurgical Hospital $(n=5)$, and Asanogawa General Hospital $(n=6)$. Although this series included previously published cases, databases were revised for this study by adding further follow-up data (UPMC, ${ }^{11}$ UVA,,${ }^{20}$ and Yokohama Rosai Hospital $^{13}$ ). There were 80 patients (36 male, 44 female) with 335 hemangioblastomas associated with von HippelLindau disease (VHL) and 106 patients (58 male and 48 female) with 182 sporadic hemangioblastomas. The median patient age was 38 years in the VHL group and 52 years in the sporadic hemangioblastoma group. Seventy VHL patients had prior resection of a hemangioblastoma (single surgery in 32 cases, multiple surgeries in 38). In the sporadic hemangioblastoma group, 86 patients had prior resection (single surgery in 53, multiple surgeries in 33). Ten patients with VHL and 20 with sporadic hemangioblastoma did not undergo prior resection, and in their cases the tumors were diagnosed by MRI and angiography. Ten VHL patients had 2 or more hemangioblastomas at separate locations within the brain and a family history of VHL. All of the patients with sporadic hemangioblastoma had either a solitary tumor or multiple tumors adjacent to the surgical cavity; none of these patients had clinical features of VHL-related neoplasia, cysts at other anatomical sites, or a family history of VHL. The patient characteristics are shown in Table 1. A database with selected variables was created and sent to all participating centers. Participating centers reviewed the medical records of these patients, entered the data into the spreadsheet, and removed all patient identifiers from the data. These deidentified data were sent to the NAGKC data-coordinating center (UPMC).

The median duration between initial diagnosis and SRS was 50 months (mean 86 months, range 0.2-384 months). The median duration between the last surgical procedure and SRS for tumor progression of that tumor was 24 months (mean 53 months, range 0.2-384 months). Fortyeight tumors in VHL patients and 129 tumors in sporadic hemangioblastoma patients received SRS at the time of tumor progression identified by imaging. Tumor progression was defined by an increase in tumor volume at the time of follow-up MRI. Fifty tumors in VHL patients and 33 tumors in sporadic hemangioblastoma patients were treated with adjuvant SRS because of residual tumor after surgical removal. In VHL patients, new tumors were diagnosed by repeat brain imaging and were detected in brain locations previously devoid of tumors. Twenty patients were diagnosed with sporadic hemangioblastoma on the basis of imaging criteria. In all other patients with sporadic hemangioblastoma, SRS was performed for treatment of recurrent tumor or residual tumor after initial resection. No patients with sporadic hemangioblastomas had any other stigmata 
TABLE 1. Patient and treatment characteristics in 186 patients with 517 hemangioblastomas

\begin{tabular}{|c|c|c|c|}
\hline Characteristic & Sporadic & VHL & Entire Series \\
\hline No. of patients & 106 & 80 & 186 \\
\hline No. of tumors & 182 & 335 & 517 \\
\hline Median age & 52 & 38 & $47(14-89)$ \\
\hline \multicolumn{4}{|l|}{ Sex (no. of pts) } \\
\hline Male & 58 & 36 & 94 \\
\hline Female & 48 & 44 & 92 \\
\hline Prior surgery (no. of pts) & 86 & 70 & 156 \\
\hline Single & 53 & 32 & 85 \\
\hline Multiple & 33 & 38 & 71 \\
\hline \multicolumn{4}{|l|}{$\begin{array}{l}\text { Lesion characteristics at } \\
\text { presentation }\end{array}$} \\
\hline New tumor & 20 & 237 & 257 \\
\hline Residual tumor & 33 & 50 & 83 \\
\hline Recurrent tumor & 129 & 48 & 177 \\
\hline \multicolumn{4}{|l|}{ Location } \\
\hline Cerebellum & 160 & 295 & 455 \\
\hline Brainstem & 15 & 32 & 47 \\
\hline Supratentrial & 7 & 8 & 15 \\
\hline Solid & 149 & 297 & 446 \\
\hline Cystic & 33 & 38 & 71 \\
\hline \multicolumn{4}{|l|}{ Target vol $\left(\mathrm{cm}^{3}\right)$} \\
\hline Mean & 3.3 & 0.91 & 1.74 \\
\hline Median & 0.65 & 0.18 & 0.24 \\
\hline Range & - & - & $0.01-39.5$ \\
\hline \multicolumn{4}{|l|}{ Total target vol $\left(\mathrm{cm}^{3}\right)$} \\
\hline Mean & 5.71 & 2.91 & 4.51 \\
\hline Median & 2.67 & 1.44 & 1.95 \\
\hline Range & - & - & $0.06-39.5$ \\
\hline \multicolumn{4}{|l|}{ Margin dose (Gy) } \\
\hline Mean & 16.1 & 17.7 & 16.7 \\
\hline Median & 15.0 & 18.0 & 16.0 \\
\hline Range & & & $8-31.4$ \\
\hline \multicolumn{4}{|l|}{ Max dose (Gy) } \\
\hline Mean & 31.5 & 28.4 & 29.5 \\
\hline Median & 31.7 & 28.6 & 30 \\
\hline Range & - & - & $6-60$ \\
\hline
\end{tabular}

of VHL such as renal, liver, or pancreatic cysts; renal carcinoma; retinal tumors; or a family history of VHL.

In patients with VHL, 295 tumors were located in the cerebellum, 32 were in the brainstem, and 8 were in supratentorial regions. In patients with sporadic hemangioblastoma, 160 lesions were located in the cerebellum, 15 were in the brainstem, and 7 were in supratentorial regions. The patients with VHL had 38 cystic tumors and 297 solid tumors. The patients with sporadic hemangioblastoma had 33 cystic and 149 solid lesions.

Neurological symptoms were present in 48 patients with VHL (60\%) and 61 patients with sporadic hemangioblastoma (58\%). In the VHL group, 40 patients had cer- ebellar symptoms, 2 had decreased vision, 3 had diplopia, 5 had sensory dysfunction, and 1 had motor weakness. In the sporadic hemangioblastoma group, 48 patients had cerebellar symptoms, 8 had diplopia, 2 had facial numbness, 3 had facial weakness, 1 had hemifacial spasm, 3 had hearing impairment, 2 had lower cranial nerve palsy, and 2 had motor weakness.

\section{Radiosurgery Technique}

Patients underwent application of an imaging-compatible stereotactic head frame under local anesthesia supplemented by intravenous conscious sedation. Highresolution MRI or (rarely) CT was then performed. The tumor was imaged using 1- to 2-mm contrast-enhanced volume acquisition images, supplemented by $2-$ to $3-\mathrm{mm}$ T2-weighted scans. The SRS target was defined as the contrast-enhanced tumor volume including the cyst wall. Radiosurgery was performed with the Model U, B, C, or 4-C Leksell Gamma Knife (Elekta Inc.). The median target volume was $0.2 \mathrm{~cm}^{3}$ (mean $0.9 \mathrm{~cm}^{3}$ ) in patients with VHL and $0.7 \mathrm{~cm}^{3}$ (mean $3.3 \mathrm{~cm}^{3}$ ) in patients with sporadic hemangioblastomas. The median tumor diameter was $7 \mathrm{~mm}$ (mean $12 \mathrm{~mm}$ ) in patients with VHL and 11 $\mathrm{mm}$ (mean $18 \mathrm{~mm}$ ) in patients with sporadic hemangioblastomas. The median margin dose was $18 \mathrm{~Gy}$ (mean 17.7 Gy) in patients with VHL and 15 Gy (mean 16.1 Gy) in patients with sporadic hemangioblastomas.

All patients were evaluated by MRI at intervals of 3-6 months after SRS. All living patients had a minimum of 6 months' follow-up. The follow-up MR images were compared with the intraoperative images, and tumor dimensions were measured in the axial, sagittal, and coronal planes. A volume was then roughly calculated by multiplying the left-right $(\mathrm{X})$, anterior-posterior $(\mathrm{Y})$, and superior-inferior $(Z)$ dimensions and dividing this number by 2. A complete response was defined as the complete disappearance of enhancing or nonenhancing tumor, a partial response was defined as $>25 \%$ shrinkage of the tumor volume, stable disease was defined as $\pm 25 \%$ change in tumor volume, and progressive disease was defined as $>$ $25 \%$ increase in volume of the tumor.

For statistical analysis we constructed Kaplan-Meier plots for survival, the rate of developing a new tumor or recurrences of residual tumor from the original tumor, and local tumor control rate using the dates of diagnosis, first SRS, follow-up MRI studies, and death or last follow-up. Univariate analysis was performed on the Kaplan-Meier curves using the log-rank test with $p<0.05$ considered significant. The Mann-Whitney U-test was used to analyze the relationship between the tumor volume of sporadic and VHL-associated hemangioblastomas. Standard statistical processing software (SPSS, version 20.0) was used.

\section{Results}

\section{Patient Survival}

At the conclusion of this assessment, 157 (84\%) patients were alive and 29 patients (14\%) had died. The median follow-up after initial diagnosis was 9.5 years (mean 12.7 years, range $0.5-20$ years). The overall survival from initial diagnosis was $97 \%$ at 3 years, $95 \%$ at 5 years, and $87 \%$ 
at 10 years. The median follow-up after SRS was 5 years (mean 5.5 years, range $0.5-18$ years). The overall survival after SRS in the entire series was $94 \%$ at 3 years, $90 \%$ at 5 years, and $74 \%$ at 10 years. Ten of 80 patients with VHLassociated hemangioblastomas were dead at the time of the last assessment. Three died due to SRS-treated tumor progression, 3 died due to tumor dissemination, 2 died due to VHL-related renal cell carcinomas, and 2 died of unknown causes. The overall survival after SRS in patients with VHL-associated hemangioblastomas was $97 \%$ at 3 years, $82 \%$ at 5 years, and $77 \%$ at 10 years (Fig. 1). Nineteen of 106 patients with sporadic hemangioblastomas were dead at the time of the last assessment. Two died due to progression of an SRS-treated tumor, 2 died due to tumor dissemination, 1 died due to adverse radiation effects (ARE), 6 died of cardiac and/or pulmonary disease, and 8 died of unknown causes. The overall survival after SRS in patients with sporadic hemangioblastomas was $91 \%$ at 3 years, $88 \%$ at 5 years, and $71 \%$ at 10 years (Fig. 1). We found no significant difference in survival between patients with VHL-associated hemangioblastomas and those with sporadic hemangioblastomas $(\mathrm{p}=0.470)($ Table 2$)$.

In univariate analysis, factors associated with longer patient survival in the entire group of 186 patients included younger age $(p=0.031)$, female sex $(p=0.018)$, absence of neurological symptoms $(\mathrm{p}=0.011)$, fewer tumors $(\mathrm{p}=$ 0.017), and higher Karnofsky Performance Status (KPS) score $(\mathrm{p}=0.013)$ (Table 2). Among patients with VHLassociated hemangioblastomas, factors associated with longer patient survival included female sex $(p=0.040)$ and fewer tumors $(p=0.045)$. Among patients with sporadic hemangioblastomas, factors associated with longer patient survival included younger age $(\mathrm{p}=0.040)$, smaller tumor volumes $(p=0.031)$, and higher KPS score $(p=0.005)$.

In multivariate analysis of the combined series, factors associated with longer patient survival were the absence of neurological deficits ( $\mathrm{p}=0.023$, HR $15.11,95 \%$ CI $1.46-157.0)$ and fewer tumors $(\mathrm{p}=0.021, \mathrm{HR}=0.21,95 \%$ CI 0.06-0.79) (Table 3). Among patients with VHL-associated hemangioblastomas, factors associated with longer survival included female sex $(\mathrm{p}=0.010$, HR $21.86,95 \%$ CI 2.092-228.4) and absence of neurological deficits ( $\mathrm{p}$ $=0.023$, HR $21.41,95 \%$ CI 1.516-302.3). Among patients with sporadic hemangioblastoma, factors associated with longer patient survival included younger age $(p=0.010$, HR 1.066, 95\% CI 1.015-1.119) and higher KPS score (p $=0.002, \mathrm{HR} 0.935,95 \% \mathrm{CI} 0.896-0.976)$.

\section{Developing New Tumors or Recurrences of Residual Tumor From the Original Tumor After Radiosurgery}

Thirty-three (41\%) of 80 VHL patients developed new untreated tumors during the observation interval. Seventeen $(16 \%)$ of 106 sporadic hemangioblastoma patients developed recurrences of residual tumor from the untreated tumors at the site of a prior tumor resection during the observation interval. The rate of developing a new tumor or recurrence of residual tumor from the original tumor was $5 \%$ at 1 year, $18 \%$ at 3 years, $33 \%$ at 5 years, and $54 \%$ at 10 years. Patients with VHL-associated hemangioblastoma were more likely to develop new tumors $(\mathrm{p}=0.002$, HR $2.59,95 \%$ CI $1.439-4.663)$.
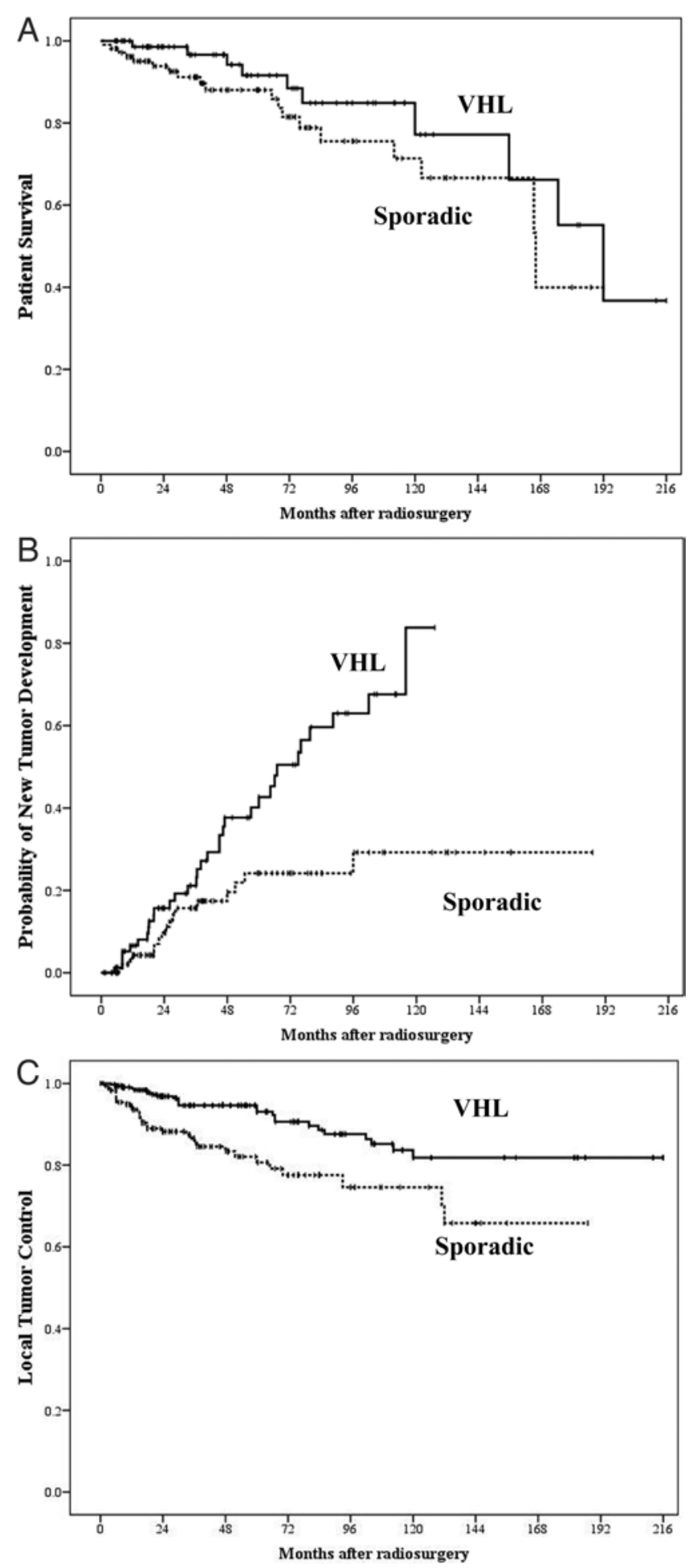

FIG. 1. A: Kaplan-Meier curves comparing the overall survival after SRS for patients with sporadic hemangioblastomas versus patients with VHL-associated hemangioblastomas. B: Kaplan-Meier curves comparing the probability of new tumor (VHL) or recurrences of residual tumor from the original tumor (sporadic) development after SRS for sporadic hemangioblastomas versus $\mathrm{VHL}$-associated hemangioblastomas. C: Kaplan-Meier curves comparing the local tumor control rate after SRS for sporadic hemangioblastomas versus VHL-associated hemangioblastomas. 


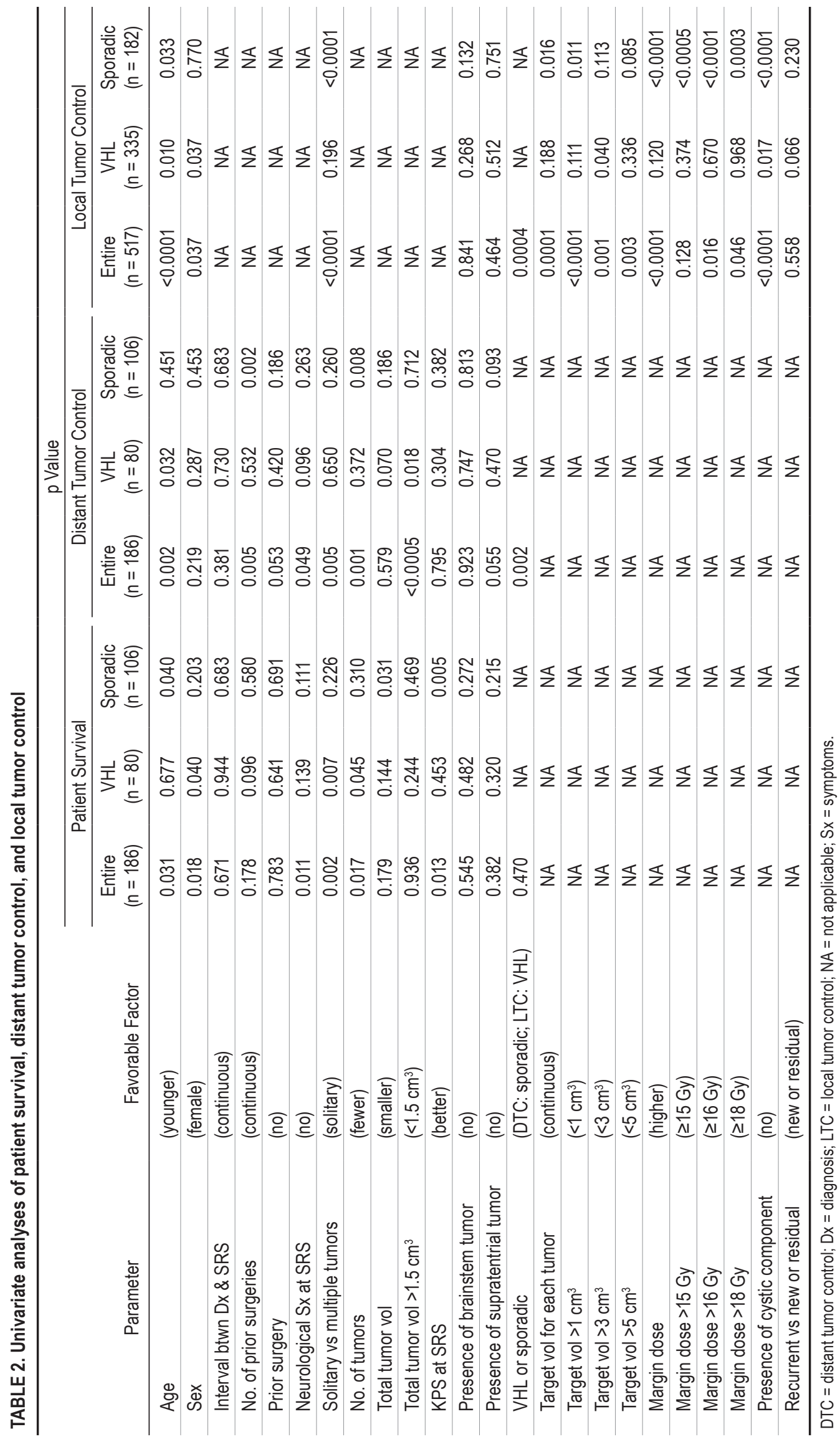


TABLE 3. Multivariate analysis of patient survival, distant tumor control, and local tumor control

\begin{tabular}{|c|c|c|c|c|c|c|c|c|c|}
\hline \multirow[b]{2}{*}{ Cohort \& Variable } & \multicolumn{3}{|c|}{ Patient Survival $(n=186)$} & \multicolumn{3}{|c|}{ Distant Tumor Control $^{*}(n=186)$} & \multicolumn{3}{|c|}{ Local Tumor Control $(n=517)$} \\
\hline & $p$ Value & $\mathrm{HR}$ & $95 \% \mathrm{Cl}$ & $\mathrm{p}$ Value & $\mathrm{HR}$ & $95 \% \mathrm{Cl}$ & p Value & $\mathrm{HR}$ & $95 \% \mathrm{Cl}$ \\
\hline \multicolumn{10}{|l|}{ Entire series } \\
\hline Age & 0.261 & NA & NA & 0.015 & 0.976 & $0.956-0.995$ & 0.323 & NA & NA \\
\hline Sex & 0.200 & NA & NA & NA & NA & NA & 0.029 & 1.891 & $1.068-3.345$ \\
\hline No. of surgeries & NA & NA & NA & 0.027 & 1.231 & $1.024-1.481$ & NA & NA & NA \\
\hline Absence of neurological Sx & 0.023 & 15.11 & $1.46-157.0$ & NA & NA & NA & NA & NA & NA \\
\hline No. of tumors & 0.021 & 0.21 & $0.06-0.79$ & 0.071 & NA & NA & $<0.0001$ & 0.289 & $0.163-0.514$ \\
\hline KPS & 0.218 & NA & NA & NA & NA & NA & NA & NA & NA \\
\hline VHL & 0.280 & NA & NA & 0.154 & NA & NA & 0.972 & NA & NA \\
\hline Tumor vol & NA & NA & NA & 0.991 & NA & NA & 0.600 & NA & NA \\
\hline Margin dose & NA & NA & NA & NA & NA & NA & $<0.0001$ & 0.890 & $0.839-0.944$ \\
\hline Cystic component & NA & NA & NA & NA & NA & NA & $<0.0001$ & 2.919 & $1.605-5.308$ \\
\hline \multicolumn{10}{|c|}{ VHL-associated hemangioma cases } \\
\hline Age & 0.149 & NA & NA & 0.032 & 0.971 & $0.945-0.997$ & 0.003 & 1.052 & $1.017-1.087$ \\
\hline Sex & 0.010 & 21.86 & $2.092-228.4$ & NA & NA & NA & 0.020 & 2.756 & $1.177-6.454$ \\
\hline No. of surgeries & NA & NA & NA & 0.283 & NA & NA & NA & NA & NA \\
\hline Absence of neurological Sx & 0.023 & 21.41 & $1.516-302.3$ & NA & NA & NA & NA & NA & NA \\
\hline No. of tumors & 0.158 & NA & NA & 0.588 & NA & NA & NA & NA & NA \\
\hline KPS & NA & NA & NA & NA & NA & NA & NA & NA & NA \\
\hline VHL & 0.084 & 0.690 & $0.453-1.051$ & 0.097 & NA & NA & 0.854 & NA & NA \\
\hline Tumor vol & NA & NA & NA & NA & NA & NA & 0.914 & NA & NA \\
\hline Margin dose & NA & NA & NA & NA & NA & NA & 0.013 & 3.010 & $1.293-8.474$ \\
\hline \multicolumn{10}{|l|}{ Sporadic hemangioma cases } \\
\hline Age & 0.010 & 1.066 & $1.015-1.119$ & 0.784 & NA & NA & 0.784 & NA & NA \\
\hline Sex & 0.117 & NA & NA & NA & NA & NA & 0.350 & NA & NA \\
\hline No. of surgeries & NA & NA & NA & 0.009 & 1.579 & $1.119-2.229$ & NA & NA & NA \\
\hline Absence of neurological Sx & 0.103 & NA & NA & NA & NA & NA & NA & NA & NA \\
\hline No. of tumors & 0.200 & NA & NA & 0.051 & 1.208 & $0.999-1.459$ & 0.013 & 0.236 & $0.076-0.734$ \\
\hline KPS & 0.002 & 0.935 & $0.896-0.976$ & NA & NA & NA & NA & NA & NA \\
\hline VHL & 0.292 & NA & NA & 0.172 & NA & NA & 0.933 & NA & NA \\
\hline Tumor vol & NA & NA & NA & NA & NA & NA & 0.002 & 0.897 & $0.838-0.960$ \\
\hline Margin dose & NA & NA & NA & NA & NA & NA & 0.039 & 2.319 & $1.044-5.151$ \\
\hline
\end{tabular}

NA = not applicable.

* No development of new tumors.

In VHL patients, the rate of new tumor development was $7 \%$ at 1 year, $21 \%$ at 3 years, $43 \%$ at 5 years, and $84 \%$ at 10 years. In univariate analysis, factors associated with higher rate of new tumor development included younger age $(\mathrm{p}$ $=0.032)$ and total tumor volume of $\geq 1.5 \mathrm{~cm}^{3}(\mathrm{p}=0.018)$ (Table 2). The number of prior operations and the number of tumors were not associated with the development of new tumors. In multivariate analysis, only younger age was associated with a higher rate of new tumor development $(\mathrm{p}=$ 0.032, HR 0.971, 95\% CI 0.945-0.997) (Table 3).

In patients with sporadic hemangioblastoma, the rate of developing a recurrence of residual tumor from the original tumor was $4 \%$ at 1 year, $16 \%$ at 3 years, $24 \%$ at 5 years, and $29 \%$ at 10 years. In univariate analysis factors associated with higher rate of developing a recurrence of residual tumor from the original tumor included larger number of prior operations $(\mathrm{p}=0.002)$ and a larger number of tumors $(\mathrm{p}=0.008)$ (Table 2). Patient age and total tumor volume was not associated with an increased risk of recurrence of residual tumor from the original tumor. In multivariate analysis, only a larger number of prior surgical procedures was associated with a higher rate of developing a recurrence of residual tumor from the original tumor $(\mathrm{p}=0.09$, HR 1.579, 95\% CI 1.119-2.229) (Table 3).

\section{Local Tumor Control}

Of 335 VHL-associated hemangioblastomas, 134 (40\%) decreased in size and 175 (52\%) were unchanged in size. Of 182 lesions in patients with 182 sporadic hemangioblastoma, $72(40 \%)$ decreased in size and $79(43 \%)$ were unchanged in size. Tumor regression was noted in an increasing number of patients whose follow-up extended beyond 
2 years $(\mathrm{p}<0.0001)$. Twenty-six $(8 \%)$ of 335 VHL-associated hemangioblastomas and $31(17 \%)$ of 182 sporadic hemangioblastomas showed progression despite SRS. The local tumor control rate in the entire group of 186 patients was $92 \%$ at 3 years, $89 \%$ at 5 years, and $79 \%$ at 10 years. In univariate analysis, factors associated with an improved local tumor control rate included VHL-associated hemangioblastoma ( $\mathrm{p}=0.0004$, HR 2.54, 95\% CI 1.51-4.30), solid tumor ( $\mathrm{p}<0.0001$, HR 3.54, 95\% CI 1.99-6.28), smaller tumor volume $(\mathrm{p}=0.0001, \mathrm{HR} 1.08,95 \%$ CI $1.04-1.12)$ and higher margin dose $(\mathrm{p}<0.0001, \mathrm{HR} 0.88,95 \%$ CI 0.84-0.93) (Table 2).

In VHL patients, the local tumor control rate after SRS was $99 \%$ at 1 year, $95 \%$ at 3 years, $93 \%$ at 5 years, and $82 \%$ at 10 years. In univariate analysis, factors associated with an improved local tumor control rate included younger age $(p=0.010)$, female sex $(p=0.037)$, tumor volume $<3 \mathrm{~cm}^{3}$ (the average tumor diameter of a tumor with a volume of $3 \mathrm{~cm}^{3}$ is $\left.18 \mathrm{~mm}\right)(\mathrm{p}=0.040)$, and solid tumor appearance on imaging ( $p=0.017$ ) (Fig. 2) (Table 2). In multivariate analysis, factors associated with an improved local tumor control rate included younger age $(\mathrm{p}=0.003$, HR 1.052 , $95 \%$ CI 1.017-1.087), female sex $(\mathrm{p}=0.020$, HR 2.756, $95 \%$ CI 6.454), and solid tumor appearance ( $\mathrm{p}=0.013$, HR 3.010, 95\% CI 1.293-8.474) (Table 3).

In sporadic hemangioblastoma patients, the local tumor control rate after SRS was $94 \%$ at 1 year, $87 \%$ at 3 years, $81 \%$ at 5 years, and $75 \%$ at 10 years. In univariate analysis, factors associated with local tumor control rate included younger age $(p=0.033)$, solitary tumor $(p<0.0001)$, smaller tumor volume $(p=0.016)$, higher margin dose $(p$ $<0.0001)$, and solid tumor appearance $(\mathrm{p}<0.0001)$ (Fig. 2) (Table 3).

The mean tumor volume of VHL-associated hemangioblastomas was $0.9 \mathrm{~cm}^{3}$ (average diameter of $12 \mathrm{~mm}$ ) compared with sporadic hemangioblastomas, which had a mean volume of $3.3 \mathrm{~cm}^{3}$ (average diameter of $18 \mathrm{~mm}$ ). At the time of SRS, sporadic hemangioblastomas were significantly larger than VHL-associated hemangioblastomas ( $p<0.0001)$. We suspect that growing VHL-associated hemangioblastomas are recognized sooner because of close MRI follow-up, which in turn prompts earlier intervention. We believe that small, asymptomatic hemangioblastomas, as are often noted in VHL patients, are best observed until documented growth is confirmed by imaging or symptoms develop.

In the entire series, the 5-year local tumor control rate in patients who underwent SRS for recurrent tumors was $86 \%$; in patients with residual tumors, it was $87 \%$; and in VHL patients with new tumors or sporadic hemangioblastoma patients with recurrence of residual tumor from the original tumor, it was $90 \%$. There was no difference in local tumor control rate in patients who underwent SRS for residual, recurrent, or new tumors (VHL) or recurrences of residual tumor from the original tumor (sporadic) $(\mathrm{p}$ $=0.558)$ (Table 2). Similarly, in both patients with VHLassociated tumors and patients with sporadic tumors we found no difference in the local tumor control rate between patients with recurrent, residual, or new tumors (VHL) or recurrences of residual tumor from the original tumor (sporadic).
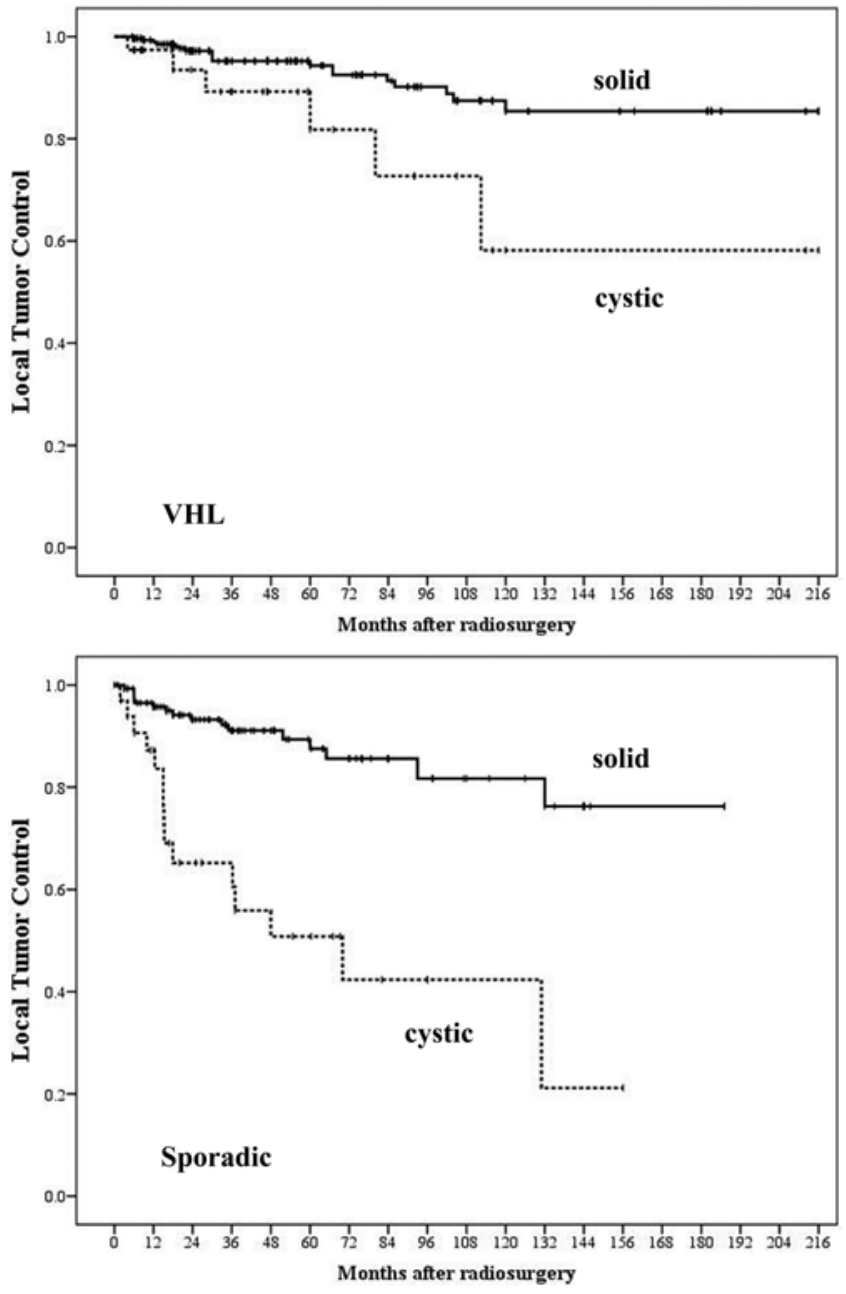

FIG. 2. Upper: Kaplan-Meier curves comparing local tumor control rates after SRS for solid versus cystic tumors in patients with sporadic hemangioblastomas. Lower: Kaplan-Meier curves comparing local tumor control rates after SRS for solid versus cystic tumors in patients with VHL-related hemangioblastomas.

\section{Adverse Radiation Effects}

A total of 13 patients developed ARE. In 10 cases, the ARE were managed successfully with oral steroids. One patient required placement of a ventriculoperitoneal shunt due to hydrocephalus 14 months after SRS. One patient with suspected ARE underwent cyst drainage 6 months after SRS. One patient with refractory peritumoral edema died 1 month after SRS (tumor volume $28.9 \mathrm{~cm}^{3}$, margin dose $10 \mathrm{~Gy})$.

\section{Additional Management After Radiosurgery}

Fifteen patients (19\%) with VHL-associated hemangioblastomas and 23 patients (22\%) with sporadic hemangioblastomas required additional treatment for treated tumor progression (Table 4). Twenty-four patients (30\%) with VHL-associated hemangioblastomas and 13 patients (12\%) with sporadic hemangioblastomas required additional treatment for new tumors (VHL) or recurrences of residual tumor from the original tumor (sporadic). 
TABLE 4. Options for additional treatment for patients with hemangioblastomas treated with SRS

\begin{tabular}{|c|c|c|c|c|}
\hline \multirow[b]{2}{*}{ Option } & \multicolumn{2}{|r|}{ Sporadic } & \multicolumn{2}{|r|}{$\mathrm{VHL}$} \\
\hline & $\begin{array}{l}\text { No. of } \\
\text { Patients }\end{array}$ & $\begin{array}{c}\text { Time btwn SRS \& } \\
\text { Additional Treatment (mos) }\end{array}$ & $\begin{array}{c}\text { No. of } \\
\text { Patients }\end{array}$ & $\begin{array}{c}\text { Time btwn SRS \& } \\
\text { Additional Treatment (mos) }\end{array}$ \\
\hline Repeat SRS for treated tumor progression & $4(4 \%)$ & Median, 68; range, 36-131 & $2(3 \%)$ & 13,102 \\
\hline Resection for treated tumor progression & $14(13 \%)$ & Median, 14; range, 3-60 & $8(10 \%)$ & Median, 44; range, 12-121 \\
\hline Cyst aspiration for treated tumor progression & $5(5 \%)$ & Median, 43; range, 15-73 & $5(6 \%)$ & Median, 60; range, 4-86 \\
\hline SRS for new (VHL) or recurrent (sporadic) tumors & $10(9 \%)$ & Median, 34; range, 8-103 & $13(16 \%)$ & Median, 55; range, 8-153 \\
\hline Resection for (VHL) or recurrent (sporadic) tumors & $2(2 \%)$ & 8,49 & $10(13 \%)$ & Median, 44; range, 8-144 \\
\hline $\begin{array}{l}\text { Cyst aspiration for (VHL) or recurrent (sporadic) } \\
\text { tumors }\end{array}$ & $1(1 \%)$ & 51 & $1(1 \%)$ & 109 \\
\hline
\end{tabular}

\section{Discussion}

Sporadic hemangioblastomas occur predominantly in the cerebellum, whereas VHL-associated hemangioblastomas may develop in the cerebellum, brainstem, or spinal cord. Whether hemangioblastomas arise sporadically or in association with VHL, they are histologically identical and are associated with mutations or deletions of the VHL gene. ${ }^{8}$ Although hemangioblastomas are histologically benign tumors that generally progress slowly, eventual tumor growth, cystic expansion, and peritumoral edema may lead to neurological morbidity or mortality. ${ }^{26}$ In this study, 10 patients with VHL-associated hemangioblastomas died3 from treated tumor progression, 3 from distant tumor dissemination, 2 from VHL-related renal cell carcinomas, and 2 from unknown causes. Nineteen patients with sporadic hemangioblastomas died, 2 from treated tumor progression, 2 from tumor dissemination, 1 from adverse radiation effects, 6 from other systemic cardiac or pulmonary disease, and 8 from unknown causes. Patients with VHL-associated hemangioblastomas were more likely to die than patients with sporadic hemangioblastomas $(\mathrm{p}=$ 0.039 ).

Ammerman et al. ${ }^{1}$ described the long-term natural history of VHL-associated hemangioblastomas. They found that these tumors tend to exhibit a saltatory growth pattern characterized by periods of growth (over an average of $13 \pm 15$ months in their case series) followed by periods of dormancy (for an average of $25 \pm 19$ months). Despite measurable growth of almost all hemangioblastomas (in $97 \%$ of patients), only $41 \%$ of their patients became symptomatic. Forty-five percent of the hemangioblastomas that eventually produced symptoms were present at the time of the initial MRI study. In the present series, $40 \%$ of VHL-associated and sporadic hemangioblastomas treated by SRS decreased in size during a median of 5 years of follow-up. After SRS, gradual tumor regression was noted more frequently as follow-up extended beyond 2 years. In this series, all treated patients had SRS for either newly developed tumors or tumors found to have progressed during serial imaging evaluations. Tumor control was defined as tumor volume regression or prevention of additional tumor growth, a convention that has been widely applied in the analysis of SRS results for other benign brain tumors, such as vestibular schwannomas and meningiomas. This retrospective study did not evaluate the alternative option of additional observation of tumors found to recur or to develop during the course of serial imaging evaluations. SRS provides an alternative to invasive surgical removal or continued observation in such patients.

\section{Resection}

When hemangioblastomas are located in critical areas, complete resection may be difficult. Jagannathan et al. ${ }^{7}$ reported on 80 hemangioblastoma patients (who underwent 126 resections for 164 cerebellar hemangioblastomas) and noted no tumor recurrences at an average of 5 years after surgery. In contrast, subtotal resection is associated with a high risk of tumor recurrence. Conway et al. ${ }^{5}$ reported on 40 hemangioblastoma patients who underwent partial resection and noted progression in 8 patients $(20 \%)$ and additional morbidity in 6 patients $(15 \%)$. Wang et al. ${ }^{27}$ reported on 39 hemangioblastoma patients who underwent radical resection. After surgery, the condition of $11 \mathrm{pa}-$ tients deteriorated and 2 patients died.

\section{Stereotactic Radiosurgery}

While resective surgery has a major role in the treatment of symptomatic hemangioblastomas, the role of stereotactic radiosurgery (SRS) has been less well defined. SRS is applied most commonly for residual tumors that were not completely removed or for patients with deepseated tumors that are considered to have excessive risks for microsurgery. Wang et al. ${ }^{28}$ reported on 35 patients with 93 hemangioblastomas treated with Gamma Knife SRS. The 5-year local tumor control rate was $71 \%$, and the actuarial survival rate was $83 \%$. Matsunaga et al..$^{13}$ reported on 22 patients with 67 hemangioblastomas treated with Gamma Knife SRS. The local tumor control rate after SRS was $88 \%$ at 5 years and $78 \%$ at 10 years. Sayer et al. ${ }^{20}$ reported a 5-year local tumor control rate of $74 \%$ in 14 patients with 26 hemangioblastomas. In the present series, the local tumor control rate after SRS for all hemangioblastomas was $89 \%$ at 5 years and $79 \%$ at 10 years. Tumor volumes of $<3 \mathrm{~cm}^{3}$ (average diameter of $18 \mathrm{~mm}$ ) in patients with VHL-associated hemangioblastoma were associated with an improved local tumor control rate ( $\mathrm{p}$ $=0.040$ ). Tumor volumes of $<1 \mathrm{~cm}^{3}$ (average diameter of $12 \mathrm{~mm}$ ) in patients with sporadic hemangioblastoma also were associated with an improved local tumor control rate $(\mathrm{p}=0.011)($ Table 2$)$. 
Kano et al. ${ }^{11}$ reported that tumor volume played a role in local tumor control rates. In the present study, margin doses of $\geq 15,16$, and 18 Gy in patients with sporadic hemangioblastoma were associated with improved local tumor control rates (Table 2). In this current experience, the margin dose delivered to patients with VHL-associated hemangioblastomas was not associated with PFS. We acknowledge that both continued observation and surgical removal represent alternative management options in patients with newly developed or recurrent tumors found to grow during serial observation. Because of the high likelihood of continued growth of recurrent or newly diagnosed tumors, SRS was used early after detection, when higher tumor doses with less risk of adverse radiation effects are feasible.

\section{Von Hippel-Lindau Disease}

In the present series, patients with VHL-associated hemangioblastomas had a much greater chance of developing a new tumor or tumor recurrence but still had better local tumor control (Table 2). Asthagiri et al. ${ }^{2}$ reported on 20 patients with 44 VHL-associated hemangioblastomas who were treated with Gamma Knife $(n=8)$ or Linacbased SRS $(n=12)$. The local tumor control rate after SRS in patients with VHL-associated hemangioblastomas was $83 \%$ at 5 years and $61 \%$ at 10 years. In the present series, the local tumor control rate after SRS was $93 \%$ at 5 years and $82 \%$ at 10 years. The local tumor control rate in patients with sporadic hemangioblastomas was $81 \%$ at 5 years and $75 \%$ at 10 years. Target volumes in patients with sporadic hemangioblastomas (mean $3.3 \mathrm{~cm}^{3}$ ) tended to be significantly larger than in patients with VHL-associated hemangioblastomas (mean $0.9 \mathrm{~cm}^{3}$ ) (Table 1). We suspect that growing VHL-associated hemangioblastomas are recognized sooner because of close MRI follow-up, prompting earlier intervention. We believe that most small, asymptomatic hemangioblastomas, detected in VHL patients warrant observation until additional growth or clinical symptoms emerge.

\section{Detection of New Tumors After Radiosurgery}

Patients with VHL frequently harbor more than one hemangioblastoma, and new tumors often develop over their life span. ${ }^{1}$ Richard et al. ${ }^{19}$ reported that VHL disease was diagnosed in more than $30 \%$ of patients with newly discovered hemangioblastomas. In the present series 33 of 80 patients with VHL-associated hemangioblastomas developed new tumors at a median of 67 months. The rate of developing a new tumor in patients with VHL-associated hemangioblastomas was $7 \%$ at 1 year, $21 \%$ at 3 years, $43 \%$ at 5 years, and $84 \%$ at 10 years (Fig. 1). Patients with sporadic hemangioblastoma developed recurrent tumors adjacent to the surgical cavity while patients with VHLassociated hemangioblastomas more frequently developed remote new tumor progression. Patients with VHL-associated hemangioblastomas should be continuously followed with MRI, and the options of further observation, resection, stereotactic cyst aspiration, or SRS should be evaluated when a new tumor is detected. In this series, a single patient died 1 month after SRS for a large volume tumor. Since the risk of adverse radiation effects is greater in pa- tients with larger tumors, resection should be the first-line option for these patients if feasible.

\section{Cystic and Solid Tumors}

In previous reports, the outcomes of SRS were less favorable for patients with cystic hemangioblastomas. Matsunaga et al. ${ }^{13}$ reported that 6 of 13 cystic hemangioblastomas were ultimately not controlled by SRS. Kano et al. ${ }^{11}$ reported that the local tumor control rate for cystic hemangioblastomas was $76 \%$ at 3 years and $61 \%$ at 5 years. In the present study, hemangioblastomas with cystic components had significantly worse local tumor control rates (VHL, $p$ $=0.017$; sporadic, $\mathrm{p}<0.0001)$. Eleven patients with cystic hemangioblastomas (VHL, $n=6$; sporadic, $n=5$ ) required resection because of cyst enlargement. Ten patients with hemangioblastomas (VHL, $n=5$; sporadic, $n=5$ ) required stereotactic cyst aspiration for treated tumor progression. Since SRS does not quickly reduce mass effect, it may not be effective for treating large symptomatic cystic hemangioblastomas. In selected patients, stereotactic cyst aspiration followed by SRS of the remaining mass may enhance local tumor control.

\section{Weaknesses of this Study}

Limitations of this study include the inherent differences in patient selection and treatment afforded by a retrospective, international multicenter study. We acknowledge that selection bias caused by treatment heterogeneity may have affected the results of the present study. In addition, we were unable to determine treatment benefit compared with observation in patients with less than 12 months of followup. This study did not evaluate the alternative management option of continued observation. All patients underwent SRS for treatment of newly diagnosed tumors or because of serial growth of tumors under observation. In a future study, we anticipate performing an analysis of outcomes for hemangioblastoma patients who undergo repeat SRS. Finally, neuroimaging and radiosurgical techniques have changed over the years. These changes have likely led to earlier detection of residual or recurrent hemangioblastoma following resection and have facilitated improvements in radiosurgical delivery. During the long period of this study, increasing experience with dose-volume relationships and tumor dose conformality and selectivity, improved treatment planning, and increasingly sophisticated MRI studies have gradually expanded our knowledge and improved SRS techniques and outcomes.

\section{Conclusions}

SRS is an important tool in selected patients with hemangioblastomas, and it is associated with a satisfactory tumor control rate as well as relatively low risk of adverse radiation effects. SRS can be applied for treatment of growing residual tumors, progressive deep-seated tumors that are high risk for microsurgery, new tumors in VHL patients, and recurrences of residual tumor from the original tumor that progress under serial observation in sporadic hemangioblastoma patients. In such cases, this study found that tumor regression or prevention of further tumor growth was achieved in 79\%-92\% of patients. 


\section{Acknowledgment}

We thank Gillian Harrison, MD (New York University Langone Medical Center), for her assistance with data collection.

\section{References}

1. Ammerman JM, Lonser RR, Dambrosia J, Butman JA, Oldfield $\mathrm{EH}$ : Long-term natural history of hemangioblastomas in patients with von Hippel-Lindau disease: implications for treatment. J Neurosurg 105:248-255, 2006

2. Asthagiri AR, Mehta GU, Zach L, Li X, Butman JA, Camphausen KA, et al: Prospective evaluation of radiosurgery for hemangioblastomas in von Hippel-Lindau disease. Neuro Oncol 12:80-86, 2010

3. Chakraborti PR, Chakrabarti KB, Doughty D, Plowman PN: Stereotactic multiple are radiotherapy. IV-Haemangioblastoma. Br J Neurosurg 11:110-115, 1997

4. Chang SD, Meisel JA, Hancock SL, Martin DP, McManus M, Adler JR Jr: Treatment of hemangioblastomas in von HippelLindau disease with linear accelerator-based radiosurgery. Neurosurgery 43:28-35, 1998

5. Conway JE, Chou D, Clatterbuck RE, Brem H, Long DM, Rigamonti D: Hemangioblastomas of the central nervous system in von Hippel-Lindau syndrome and sporadic disease. Neurosurgery 48:55-63, 2001

6. Filling-Katz MR, Choyke PL, Oldfield E, Charnas L, Patronas NJ, Glenn GM, et al: Central nervous system involvement in Von Hippel-Lindau disease. Neurology 41:41-46, 1991

7. Jagannathan J, Lonser RR, Smith R, DeVroom HL, Oldfield $\mathrm{EH}$ : Surgical management of cerebellar hemangioblastomas in patients with von Hippel-Lindau disease. J Neurosurg 108:210-222, 2008

8. Kanno H, Kondo K, Ito S, Yamamoto I, Fujii S, Torigoe S, et al: Somatic mutations of the von Hippel-Lindau tumor suppressor gene in sporadic central nervous system hemangioblastomas. Cancer Res 54:4845-4847, 1994

9. Kano H, Iqbal FO, Sheehan J, Mathieu D, Seymour ZA, Niranjan A, et al: Stereotactic radiosurgery for chordoma: a report from the North American Gamma Knife Consortium. Neurosurgery 68:379-389, 2011

10. Kano H, Kondziolka D, Mathieu D, Stafford SL, Flannery TJ, Niranjan A, et al: Stereotactic radiosurgery for intractable cluster headache: an initial report from the North American Gamma Knife Consortium. J Neurosurg 114:1736-1743, 2011

11. Kano H, Niranjan A, Mongia S, Kondziolka D, Flickinger JC, Lunsford LD: The role of stereotactic radiosurgery for intracranial hemangioblastomas. Neurosurgery 63:443-451, 2008

12. Maher ER, Yates JR, Ferguson-Smith MA: Statistical analysis of the two stage mutation model in von Hippel-Lindau disease, and in sporadic cerebellar haemangioblastoma and renal cell carcinoma. J Med Genet 27:311-314, 1990

13. Matsunaga S, Shuto T, Inomori S, Fujino H, Yamamoto I: Gamma knife radiosurgery for intracranial haemangioblastomas. Acta Neurochir (Wien) 149:1007-1013, 2007

14. Miyagami M, Katayama Y, Nakamura S: Clinicopathological study of vascular endothelial growth factor (VEGF), p53, and proliferative potential in familial von Hippel-Lindau disease and sporadic hemangioblastomas. Brain Tumor Pathol 17:111-120, 2000

15. Neumann HP, Berger DP, Sigmund G, Blum U, Schmidt D, Parmer RJ, et al: Pheochromocytomas, multiple endocrine neoplasia type 2, and von Hippel-Lindau disease. N Engl J Med 329:1531-1538, 1993

16. Pan L, Wang EM, Wang BJ, Zhou LF, Zhang N, Cai PW, et al: Gamma knife radiosurgery for hemangioblastomas. Stereotact Funct Neurosurg 70 (Suppl 1):179-186, 1998
17. Park YS, Chang JH, Chang JW, Chung SS, Park YG: Gamma knife surgery for multiple hemangioblastomas. J Neurosurg 102 Suppl:97-101, 2005

18. Patrice SJ, Sneed PK, Flickinger JC, Shrieve DC, Pollock BE, Alexander E III, et al: Radiosurgery for hemangioblastoma: results of a multiinstitutional experience. Int J Radiat Oncol Biol Phys 35:493-499, 1996

19. Richard S, Campello C, Taillandier L, Parker F, Resche F: Haemangioblastoma of the central nervous system in von Hippel-Lindau disease. J Intern Med 243:547-553, 1998

20. Sayer FT, Nguyen J, Starke RM, Yen CP, Sheehan JP: Gamma knife radiosurgery for intracranial hemangioblastomasoutcome at 3 years. World Neurosurg 75:99-105, 2011

21. Sheehan JP, Tanaka S, Link MJ, Pollock BE, Kondziolka $\mathrm{D}$, Mathieu D, et al: Gamma Knife surgery for the management of glomus tumors: a multicenter study. J Neurosurg 117:246-254, 2012

22. Smalley SR, Schomberg PJ, Earle JD, Laws ER Jr, Scheithauer BW, O'Fallon JR: Radiotherapeutic considerations in the treatment of hemangioblastomas of the central nervous system. Int J Radiat Oncol Biol Phys 18:1165-1171, 1990

23. Sora S, Ueki K, Saito N, Kawahara N, Shitara N, Kirino T: Incidence of von Hippel-Lindau disease in hemangioblastoma patients: the University of Tokyo Hospital experience from 1954-1998. Acta Neurochir (Wien) 143:893-896, 2001

24. Sung DI, Chang CH, Harisiadis L: Cerebellar hemangioblastomas. Cancer 49:553-555, 1982

25. Tago M, Terahara A, Shin M, Maruyama K, Kurita H, Nakagawa K, et al: Gamma knife surgery for hemangioblastomas. J Neurosurg 102 Suppl:171-174, 2005

26. Wanebo JE, Lonser RR, Glenn GM, Oldfield EH: The natural history of hemangioblastomas of the central nervous system in patients with von Hippel-Lindau disease. J Neurosurg 98:82-94, 2003

27. Wang C, Zhang J, Liu A, Sun B: Surgical management of medullary hemangioblastoma. Report of 47 cases. Surg Neurol 56:218-227, 2001

28. Wang EM, Pan L, Wang BJ, Zhang N, Zhou LF, Dong YF, et al: The long-term results of gamma knife radiosurgery for hemangioblastomas of the brain. J Neurosurg 102 Suppl: 225-229, 2005

\section{Author Contributions}

Conception and design: Kano, Lunsford. Acquisition of data: Kano, Shuto, Iwai, Sheehan, Yamamoto, McBride, Sato, Serizawa, Yomo, Moriki, Kohda, Young, Suzuki, Kenai, Duma, Kikuchi, Mathieu, Akabane, Nagano. Analysis and interpretation of data: Kano. Drafting the article: Kano, Lunsford. Critically revising the article: all authors. Reviewed submitted version of manuscript: all authors. Approved the final version of the manuscript on behalf of all authors: Kano. Statistical analysis: Kano. Study supervision: Kano.

\section{Supplemental Information \\ Previous Presentation}

This study was presented and awarded the Leksell Radiosurgery Award at the 2013 Annual Meeting of the American Association of Neurological Surgeons.

\section{Correspondence}

Hideyuki Kano, Department of Neurological Surgery, University of Pittsburgh, Ste. B-400, UPMC Presbyterian, 200 Lothrop St., Pittsburgh,PA 15213. email: kanoh@upmc.edu. 